\title{
Expansion Pathology: Nanoscale Imaging of Clinical Specimens with Optical Microscopy
}

Yongxin Zhao ${ }^{1}$, Feifei Fu ${ }^{1}$ and Octavian Bucur ${ }^{2}$

${ }^{1}$ Carnegie Mellon University, Pittsburgh, Pennsylvania, United States, ${ }^{2}$ QPathology, Boston, Massachusetts, United States

In modern biology, diffraction-limited microscopy is a powerful tool to observe microscopic structures and processes of biological specimens. However, diffraction-limited microscopy is unable to resolve nanoscale configurations of biomolecules below the diffraction limit, which severely limited its capability of analyzing intricate and subtle biological/pathological changes. Recently, Expansion Microscopy (ExM) has emerged as a ground-breaking new principle for scalable, nanoscale optical imaging of biological specimens ${ }^{1,2}$. Rather than optically magnify samples, ExM works by embedding biological tissue into a water-swellable polyelectrolyte hydrogel, enzymatically homogenizing them, and then isotropically expanding the tissue-hydrogel physically in pure water. Typical ExM protocols expand tissues by $\sim 100$ folds in volume, thus enabling nanoscale optical imaging with resolution $\sim 60 \mathrm{~nm}$ using diffraction-limited microscopes ${ }^{1,2}$.

We further developed a clinically optimized form of ExM that supports nanoscale imaging of human tissue specimens fixed with formalin, embedded in paraffin, stained with hematoxylin and eosin, and/or fresh frozen. The method, called expansion pathology (ExPath), transforms clinical samples into an ExMcompatible state, then utilizes an ExM protocol with protein anchoring and mechanical homogenization steps optimized for clinical samples ${ }^{3,4}$ (Fig.1) ExPath enables $\sim 60$-nm-resolution imaging of diverse biomolecules in intact tissues using conventional diffraction-limited microscopes and standard antibody and fluorescent DNA in situ hybridization reagents. We use ExPath for optical diagnosis of kidney minimal-change disease, a process that previously required electron microscopy, and we demonstrate high-fidelity computational discrimination between early breast neoplastic lesions for which pathologists often disagree in classification. We also reported a new, fast variant, rapid expansion pathology, that can be performed on $<5$ - $\mu \mathrm{m}$-thick tissue sections, taking $<4 \mathrm{~h}$ with immunostained tissue sections and $<8 \mathrm{~h}$ with unstained specimens ${ }^{5}$ (Fig. 2). In contrast, the conventional expansion pathology, can be completed in $\sim 1 \mathrm{~d}$ with immunostained tissue sections and $2 \mathrm{~d}$ with unstained specimens. Both ExPath versions require only inexpensive, commercially available reagents and hardware commonly found in a routine pathology laboratory, thus these new techniques may enable the routine use of nanoscale imaging in pathology and clinical research.

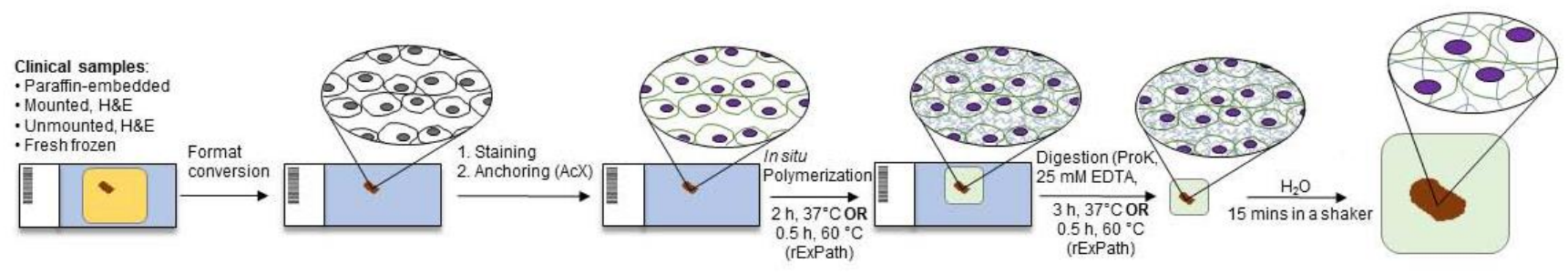

Figure 1. Schematic of ExPath/rExPath workflow. Adapted from Ref. 5. 

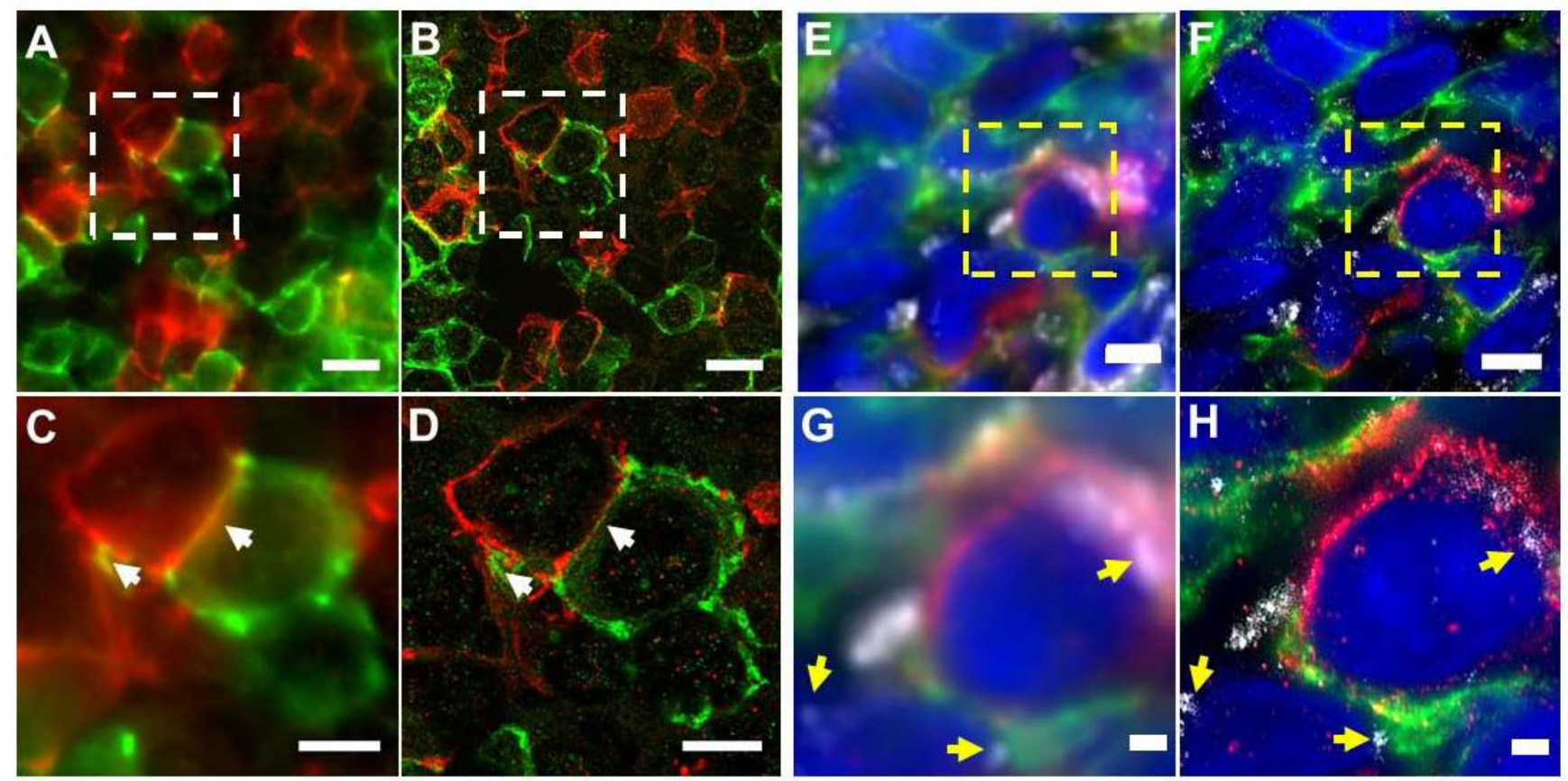

Figure 2. Rapid ExPath imaging of lymph node specimens from patients. (A) Pre-expansion image of a normal human lymph node specimen acquired with a spinning disk confocal microscope. Green, IgD; Red, CD8. (B) rExPath image of the specimen of A acquired with the same confocal microscope. Expansion factor: 4.0. (C and D) Fields of view zoomed into the corresponding areas outlined by a dashed white box in A and B, respectively. White arrows indicate examples of pre-expansion overlapped IgD and CD8 patterns being resolved after expansion. (E) Pre-expansion image of a human lymph node specimen with HIV acquired with a wide-field fluorescence microscope. Green, CD8; Red, PD-1; Grey, p24; Blue, DAPI. (F) rExPath image of the specimen of E acquired with the same microscope. Expansion factor: 4.58. ( $\mathrm{G}$ and $\mathrm{H}$ ) Corresponding fields of view zoomed into the areas outlined by a dashed yellow box in $\mathrm{E}$ and $\mathrm{F}$, respectively. Yellow arrows indicate examples of p24 being localized with sub-diffraction limit precision. Scale bar (biological scale): (A and B) $5 \mu \mathrm{m}$; (C and D) $1 \mu \mathrm{m}$; (E and F) $10 \mu \mathrm{m}$; (G and H) 2 $\mu \mathrm{m}$. Adapted from Ref. 5.

\section{References}

(1) Chen, F.; Tillberg, P. W.; Boyden, E. S. Expansion Microscopy. Science 2015, 347 (6221), 543548. https://doi.org/10.1126/science.1260088.

(2) Wassie, A. T.; Zhao, Y.; Boyden, E. S. Expansion Microscopy: Principles and Uses in Biological Research. Nat. Methods 2019, 16 (1), 33-41. https://doi.org/10.1038/s41592-018-0219-4.

(3) Zhao, Y.; Bucur, O.; Irshad, H.; Chen, F.; Weins, A.; Stancu, A. L.; Oh, E.-Y. Y.; Distasio, M.; Torous, V.; Glass, B.; Stillman, I. E.; Schnitt, S. J.; Beck, A. H.; Boyden, E. S. Nanoscale Imaging of Clinical Specimens Using Pathology-Optimized Expansion Microscopy. Nat Biotech 2017, 35 (8), 757764. https://doi.org/10.1038/nbt.3892.

(4) Klimas, A.; Bucur, O.; Njeri, B.; Zhao, Y. Nanoscopic Imaging of Human Tissue Sections via Physical and Isotropic Expansion. JoVE 2019, No. 151, e60195. https://doi.org/doi:10.3791/60195.

(5) Bucur, O.; Fu, F.; Calderon, M.; Mylvaganam, G. H.; Ly, N. L.; Day, J.; Watkin, S.; Walker, B. D.; Boyden, E. S.; Zhao, Y. Nanoscale Imaging of Clinical Specimens Using Conventional and RapidExpansion Pathology. Nat. Protoc. 2020, 15 (5), 1649-1672. https://doi.org/10.1038/s41596-020-0300-1. 